\title{
LncRNA profile of glioblastoma reveals the potential role of IncRNAs in contributing to glioblastoma pathogenesis
}

\author{
LEI HAN ${ }^{1-3}$, KAILIANG ZHANG ${ }^{1-3}$, ZHENDONG SHI $^{1-3}$, JUNXIA ZHANG ${ }^{1-3}$, JIALIN ZHU $^{1-3}$, \\ SHANJUN ZHU ${ }^{1-3}$, ANLING ZHANG ${ }^{1-3}$, ZHIFAN JIA ${ }^{1-3}$, GUANGXIU WANG $^{1-3}$, \\ SHIZHU YU ${ }^{1-3}$, PEIYU PU ${ }^{1-3}$, LUN DONG $^{4}$ and CHUNSHENG KANG ${ }^{1-3}$ \\ ${ }^{1}$ Department of Neurosurgery, Laboratory of Neuro-Oncology, Tianjin Medical University General Hospital; \\ ${ }^{2}$ Key Laboratory of Post-trauma Neuro-repair and Regeneration in Central Nervous System, Ministry of Education; \\ ${ }^{3}$ Tianjin Key Laboratory of Injuries, Variations and Regeneration of Nervous System, Tianjin 300052; ${ }^{4}$ Department \\ of Neurosurgery, Clinical Medical College of Yangzhou University, Yangzhou 225001, Jiangsu, P.R. China
}

Received December 9, 2011; Accepted January 27, 2012

DOI: 10.3892/ijo.2012.1413

\begin{abstract}
Long non-coding RNAs (lncRNAs) have recently emerged as a major class of regulatory molecules involved in a broad range of biological processes and complex diseases. Our aim was to identify important lncRNAs that might play an important role in contributing to glioblastoma (GBM) pathogenesis by conducting lncRNA and mRNA profile comparison between GBM and normal brain tissue. The differentially expressed IncRNA and mRNA profiles of the tissue between GBM patient and age-matched donor without GBM diseases were analyzed using microarrays. We propose a novel model for the identification of lncRNA-mRNA targeting relationships that combine the potential targets of the differentially expressed lncRNAs with the differentially expressed mRNA abundance data. Bioinformatic analysis of the predicted target genes (gene ontology, pathway and network analysis) was performed for further research. The lncRNA microarray reveals differentially expressed lncRNAs between GBM and normal brain tissues. In the GBM group, 654 lncRNAs were upregulated and 654 were downregulated (fold change $\geq 4.0$ or $\leq 0.25, \mathrm{P}<0.01$ ). We found 104 matched lncRNAmRNA pairs for 91 differentially expressed lncRNAs and 84 differentially expressed genes. Target gene-related pathway analysis showed significant changes in PPAR pathways in the GBM group compared with the normal brain group $(\mathrm{P}<0.05)$.
\end{abstract}

Correspondence to: Dr Lun Dong, Department of Neurosurgery, Clinical Medical College of Yangzhou University, Yangzhou 225001, Jiangsu, P.R. China

E-mail: dongluen@163.com

Dr Chunsheng Kang, Laboratory of Neuro-Oncology, Tianjin Neurological Institute, Tianjin Medical University General Hospital, Tianjin 300052, P.R. China

E-mail:kang97061@yahoo.com

Key words: glioma, long non-coding RNAs, microarray, glioma pathway, glioblastoma, mRNA, network
By further conducting lncRNA gene network analysis, we found that ASLNC22381 and ASLNC2081 were likely to play roles in the regulation of glioma signaling pathways. In conclusion, our results indicated that the IncRNA expression profile in GBM tissue was significantly altered. These results may provide important insights into the mechanisms responsible for GBM progression and pathogenesis. This study also suggests that ASLNC22381 and ASLNC20819 may play important roles via their target IGF-1 in the recurrence and malignant progression of GBM.

\section{Introduction}

Glioblastoma (GBM) is the most common and aggressive type of primary brain tumor in human $(1,2)$. In the past two decades, the molecular mechanisms, genetics and pathways to treat GBM have extensively been studied. However, the precise mechanism of GBM is unknown and its median survival rate is very low $(3,4)$. The Cancer Genome Atlas (TCGA) generated large-scale multi-dimensional datasets to catalogue cancer alterations. GBM is the first cancer studied by TCGA (5).

Large-scale analyses of full-length cDNA sequences have detected large numbers of long non-coding RNAs (lncRNAs) in human, mouse and fly. These lncRNAs have been shown to play key roles in imprinting control, cell differentiation, immune responses, human diseases, tumorigenesis and other biological processes (6). In particular, the regulatory roles of lncRNAs in the expression, activity and localization of protein-coding genes have attracted attention (7). Although an increasing number of lncRNAs are being characterized, the functions of most lncRNAs are still unknown (8). Generally, lncRNAs are as poorly conserved as the introns of coding genes and less conserved than the 5'- or 3'-untranslated regions (UTRs) of mRNAs. However, lack of conservation does not necessarily mean lack of function, as demonstrated by the very poorly conserved lncRNA Xist transcript, which plays a critical role in regulation of imprinted and random $X$ inactivation (9). The low-conservation level of lncRNAs suggests they evolve more quickly than protein-coding genes, rendering functional 
prediction by genomic comparison very difficult. Besides, functional prediction of lncRNAs is also hampered by the lack of collateral information. It has been proposed that the functional properties of lncRNAs are mainly related to their secondary structures (10). In spite of much effort, the number of lncRNAs with known functions remains scarce, and efficient prediction of lncRNA functions is still a considerable challenge.

In the past, although enormous efforts toward generating high-throughput genetic and molecular data have been made, unravelling the mechanisms of GBM remains unresolved via integrating genetic and molecular data. It is increasingly recognized that lncRNAs have emerged as an important component in the regulation of gene expression. In this study, we profiled lncRNAs and mRNAs expression in the tissue of GBM and normal brain by microarray. The differentially expressed lncRNAs were then selected to bioinformatic analyses for target prediction. To improve the accuracy of target prediction, we further combined differentially expressed mRNA with target prediction of differentially expressed lncRNAs. The predicted target genes above are subjected to bioinformatic analyses, including gene ontology analysis, pathway analysis, and network analysis. Analyzing the potential molecular markers and the possible relationship between the differentially expressed protein-coding genes and lncRNAs in GBM will help to give further insight into the pathogenesis of GBM.

\section{Materials and methods}

Sample preparation and RNA extraction. One GBM tissue from a 57-year-old female and one normal brain tissue from an agematched female donor without GBM diseases were obtained according to clinical protocols at the clinical medical college of Yangzhou University. The patient had not received prior treatment. As shown in Fig. 1, imaging and histopathological analysis revealed GBM. Following informed consent at the time of acquisition, the samples were collected, and stored at liquid nitrogen. Tissue samples were homogenized in TRIzol reagent (Invitrogen, CA). The total RNA was isolated according to the manufacturer's protocol (Invitrogen). RNA quantity and quality were measured by NanoDrop ND-1000 and RNA integrity was assessed using standard denaturing agarose gel electrophoresis (11).

Profiling of lncRNA and mRNA expression in GBM and normal brain samples

lncRNA and mRNA microarray. The Human 12x135k long noncoding RNA array was manufactured by Roche NimbleGen. Each array represented all long transcripts, both protein coding mRNAs and lncRNAs in the human genome. More than 23,000 lncRNAs were collected from the authoritative data sources including NCBI RefSeq, UCSC, RNAdb, IncRNAs from literatures and UCRs. Each transcript is represented by 1-5 probes to improve statistical confidence.

RNA labeling and array hybridization. Double-strand cDNA (ds-cDNA) was synthesized from $5 \mu \mathrm{g}$ of total RNA using an Invitrogen SuperScript ds-cDNA synthesis kit in the presence of 100 pmol oligo dT primers. ds-cDNA was cleaned and labeled in accordance with the NimbleGen Gene Expression Analysis protocol (NimbleGen Systems, Inc., Madison, WI,
USA). Microarrays were hybridized at $42^{\circ} \mathrm{C}$ for 16 to $20 \mathrm{~h}$ with $4 \mu \mathrm{g}$ of $\mathrm{Cy} 3$ labelled ds-cDNA in NimbleGen hybridization buffer/hybridization component $\mathrm{A}$ in a hybridization chamber (Hybridization System-NimbleGen Systems, Inc.). Following hybridization, washing was performed using the NimbleGen Wash Buffer kit (NimbleGen Systems, Inc.). After being washed in an ozone-free environment, the slides were scanned using the Axon GenePix 4000B microarray scanner.

Data analysis. Slides were scanned at $5 \mu \mathrm{m} /$ pixel resolution using an Axon GenePix 4000B scanner (Molecular Devices Corporation) piloted by GenePix Pro 6.0 software (Axon). Scanned images were then imported into NimbleScan software (version 2.5) for grid alignment and expression data analysis. Expression data were normalized through quantile normalization and the Robust Multichip Average (RMA) algorithm included in the NimbleScan software. The Probe level files and mRNA level files were generated after normalization. All mRNA level files were imported into Agilent GeneSpring GX software (version 11.0) for further analysis. Differentially expressed lncRNAs and mRNAs were identified through Fold Change filtering.

\section{IncRNA target prediction}

Identify the targets of differentially expressed lncRNAs via cisor trans-regulatory effects. Differentially expressed lncRNAs were selected for target prediction. Two independent algorithms were used. The first algorithm searches for target genes acting in cis. With the help of gene annotations at UCSC (http://genome. ucsc.edu/), lncRNAs and potential target genes were paired and visualized using UCSC genome browser. The genes transcribed within a $10 \mathrm{kbp}$ window upstream or downstream of lncRNAs were considered as cis target genes. The second algorithm is based on mRNA sequence complementarity and RNA duplex energy prediction, assessing the impact of lncRNA binding on complete mRNA molecules. It uses the BLAST software for first round screening. Finally, the RNAplex software was used to choose trans-acting target genes (12). RNAplex parameters were set as -e -20 .

Combine differentially expressed $m R N A$ s with target prediction of differentially expressed lncRNAs. The second step was to integrate the predicted potential lncRNA targets above with the differently expressed mRNAs in the profile (fold change $\geq 4.0$ or $\leq 0.25$ ).

Functional group analysis. The predicted target genes above were input into the Database for Annotation, Visualization and Integrated Discovery (DAVID, http://david.abcc.ncifcrf.gov/), which leveraged the Gene Ontology (GO) to identify the molecular function represented in the gene profile (13). In addition, we used the KEGG (Kyoto Encyclopedia of Genes and Genomes) database (http://www.genome.ad.jp/kegg/) and BioCarta (http:// www.biocarta. com) to analyze the roles of these target genes in the pathways (14).

Discovery of IncRNA-mRNA regulatory network. To show that the lncRNAs directly regulated the expression of targets mRNAs, we superimposed lncRNA target predictions onto the lncRNA-mRNA correlation network. The resulting network 

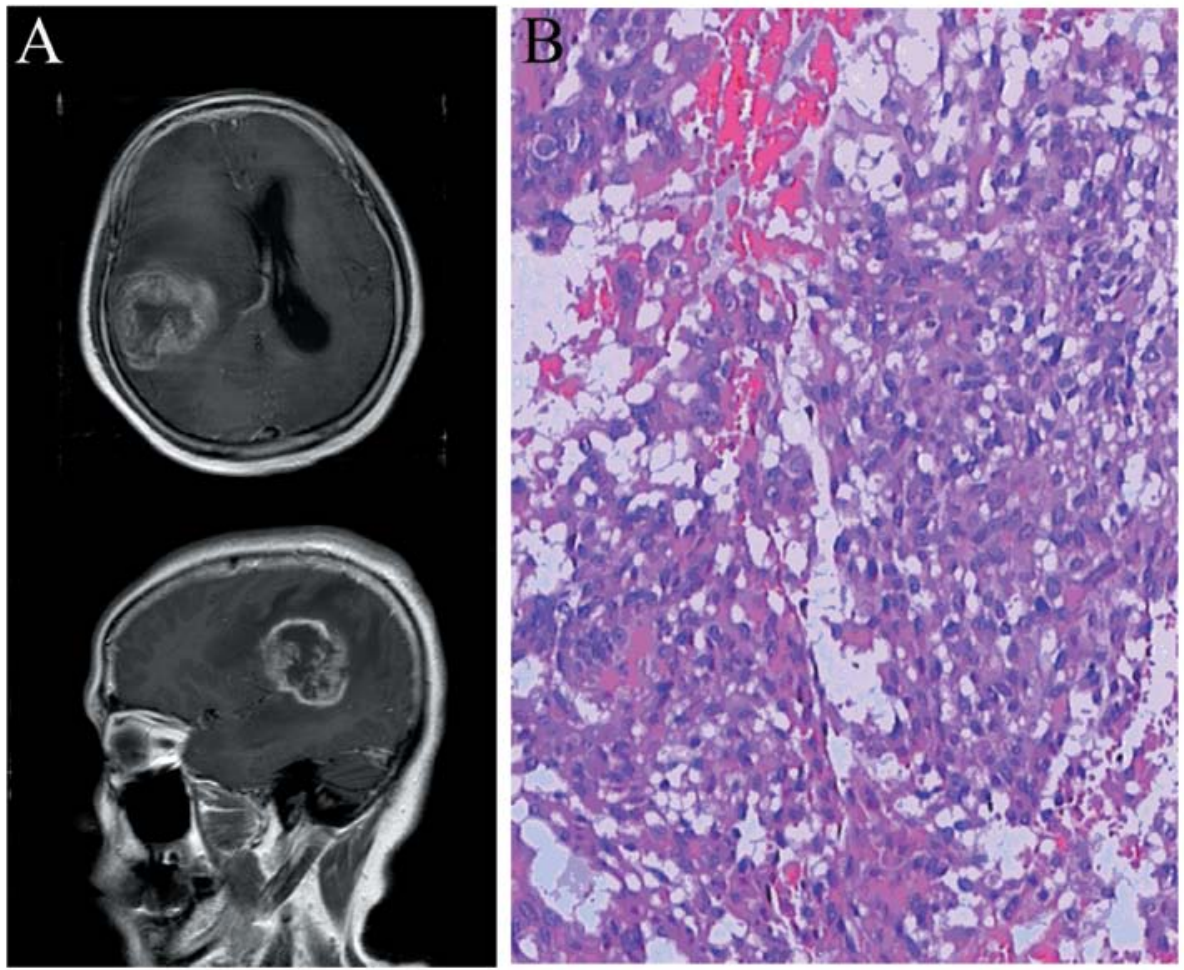

Figure 1. Clinical characteristics of a GBM patient. MRI scans (A) and histopathological features (B) of a 57-year-old female patient with GBM.
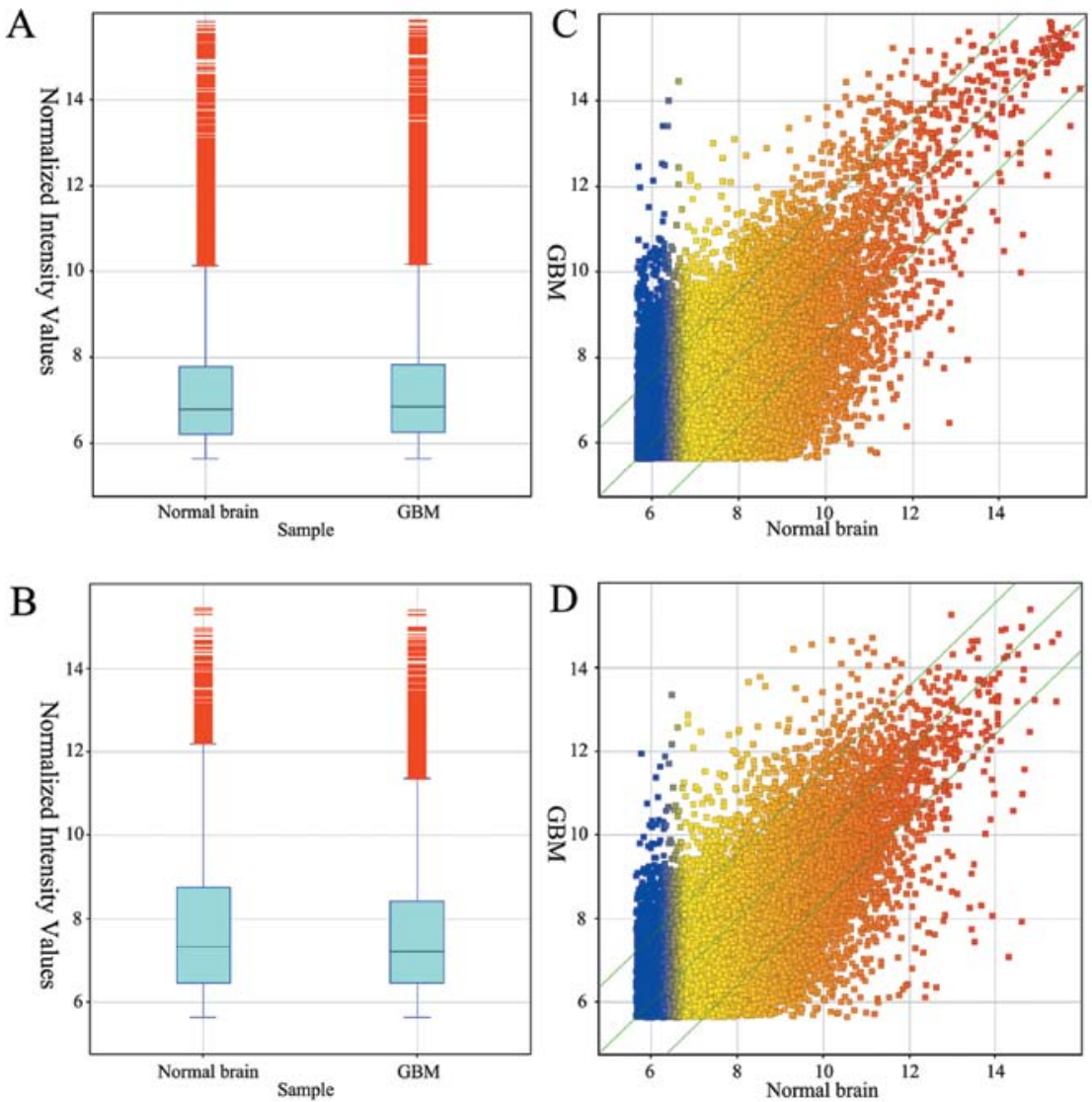

Figure 2. IncRNA and mRNA profile comparation between the GBM sample and the normal brain sample. The box plot is a convenient way to visualize the distribution of a dataset in the lncRNA (A) and mRNA (B) profiles. After normalization, the distributions of log2-ratios among the tested samples are nearly the same. The scatter-plot is used for assessing the lncRNA (C) and mRNA (D) expression variation between the GBM and normal brain compared arrays. The values of $\mathrm{X}$ and $\mathrm{Y}$ axes in the scatter-plot are averaged normalized values in each group ( $\log 2$ scaled). The green lines are fold change lines (the default fold change value given is 3.0). The lncRNAs above the top green line and below the bottom green line indicate more than 3-fold change of lncRNAs between pairs. 
A Biological Process

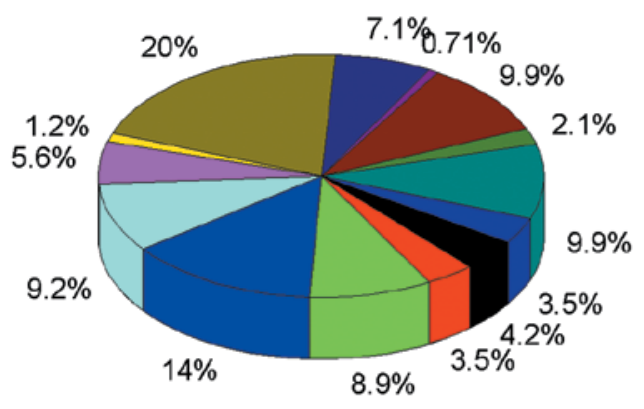

B Cellular Component

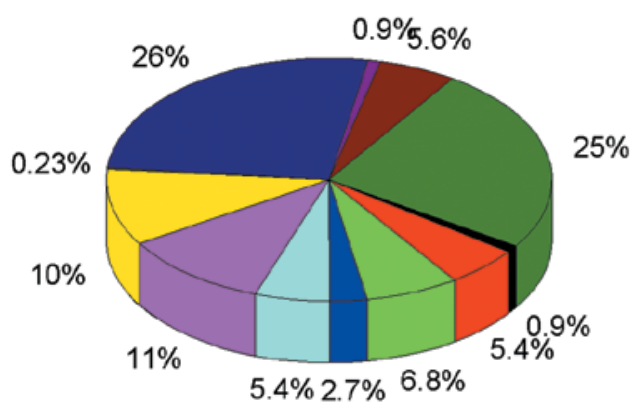

C Molecular Function

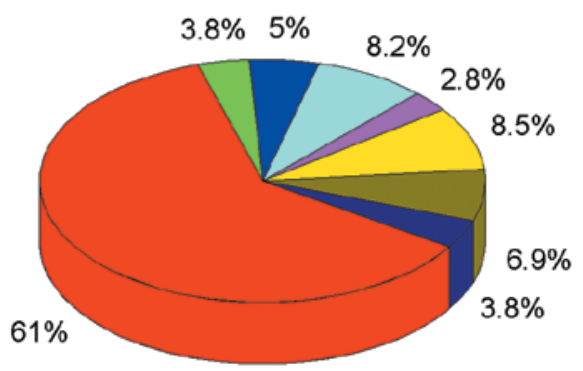

cell cycle and proliferation

stress response transport

other metabolic processes developmental processes

RNA metabolism

DNA metabolism

other biological processes

cell organization and biogenesis

cell-cell signaling

signal transduction

cell adhesion

protein metabolism

death

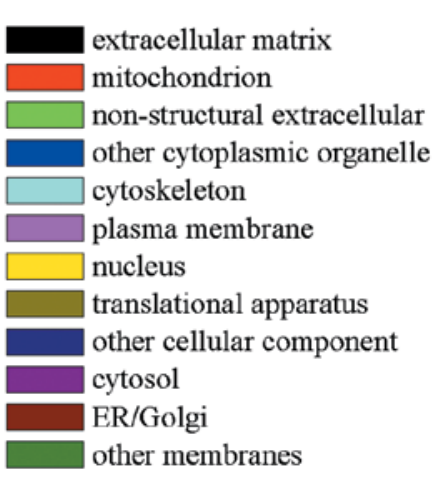

Figure 3. Gene ontology (GO) enrichment analysis for lncRNA-targets in the category of biological processes. (A) Gene Oncology analysis of lncRNA-target genes according to biological process. (B) Gene Oncology analysis of lncRNA-target genes according to cell component. (C) Gene Oncology analysis of lncRNA-target genes according to molecular function.

was defined as an IncRNA-mRNA regulatory network. A direct connection was placed from an IncRNA to an mRNA using the solid line (trans interaction) or dotted line (cis interaction).

Statistical analysis. All the results were expressed as mean \pm standard deviation. Statistical analysis was done with the Student's t-test for comparison of two groups in microarray, and analysis of variance for multiple comparisons. In both cases, differences with $\mathrm{P}<0.05$ were considered statistically significant. The statistically significance of microarray result was analyzed by fold change and the Student's t-test. False discovery rate (FDR) was calculated to correct the P-value. The threshold value we used to screen differentially expressed lncRNAs and mRNAs is a fold change $\geq 4.0$ or $\leq 0.25(\mathrm{P}<0.01)$.

\section{Results}

IncRNAs are aberrantly expressed in GBM compared with normal brain. To study the potential biological functions of lncRNAs in GBM, we determined the lncRNA and mRNA expression profile in human GBM through microarray analysis (Fig. 2). Using the authoritative data sources consisting of more than 23,000 lncRNAs, we firstly assessed the lncRNA expression profiles in the GBM and normal brain tissues. The 
Table I. Functional classification of the target genes by GO.

\begin{tabular}{|c|c|c|c|}
\hline Term & Count & $\%$ & P-value \\
\hline \multicolumn{4}{|l|}{ Biological process } \\
\hline Other metabolic processes & 59 & 13.88235 & 8.64E-05 \\
\hline Transport & 38 & 8.94118 & 0.11666 \\
\hline Death & 15 & 3.52941 & 0.12683 \\
\hline Protein metabolism & 42 & 9.88235 & 0.1408 \\
\hline Cell organization and biogenesis & 30 & 7.05882 & 0.16454 \\
\hline Developmental processes & 39 & 9.17647 & 0.20896 \\
\hline Cell cycle and proliferation & 18 & 4.23529 & 0.36535 \\
\hline Cell adhesion & 9 & 2.11765 & 0.41888 \\
\hline Stress response & 15 & 3.52941 & 0.52813 \\
\hline DNA metabolism & 5 & 1.17647 & 0.62532 \\
\hline Other biological processes & 86 & 20.23529 & 0.64087 \\
\hline Cell-cell signaling & 3 & 0.70588 & 0.81751 \\
\hline Signal transduction & 42 & 9.88235 & 0.87979 \\
\hline RNA metabolism & 24 & 5.64706 & 0.98891 \\
\hline \multicolumn{4}{|l|}{ Cellular component } \\
\hline Extracellular matrix & 4 & 0.90293 & $3.65 \mathrm{E}-01$ \\
\hline Other cytoplasmic organelle & 12 & 2.7088 & $8.64 \mathrm{E}-05$ \\
\hline Non-structural extracellular & 30 & 6.77201 & 0.11666 \\
\hline Other cellular component & 116 & 26.1851 & 0.16454 \\
\hline Cytoskeleton & 24 & 5.41761 & 0.20896 \\
\hline Other membranes & 110 & 24.8307 & 0.41888 \\
\hline Mitochondrion & 24 & 5.41761 & 0.52813 \\
\hline Nucleus & 45 & 10.15801 & 0.62532 \\
\hline Translational apparatus & 1 & 0.22573 & 0.64087 \\
\hline Cytosol & 4 & 0.90293 & 0.81751 \\
\hline ER/Golgi & 25 & 5.64334 & 0.87979 \\
\hline Plasma membrane & 48 & 10.83521 & 0.98891 \\
\hline \multicolumn{4}{|l|}{ Molecular function } \\
\hline Other molecular function & 195 & 61.12853 & $2.21 \mathrm{E}-04$ \\
\hline Transcription regulatory activity & 12 & 3.76176 & $8.21 \mathrm{E}-01$ \\
\hline Cytoskeletal activity & 22 & 6.89655 & $1.55 \mathrm{E}-06$ \\
\hline Transporter activity & 16 & 5.01567 & 0.33468 \\
\hline Kinase activity & 12 & 3.76176 & 0.46227 \\
\hline Enzyme regulator activity & 9 & 2.82132 & 0.53214 \\
\hline Nucleic acid binding activity & 27 & 8.46395 & 0.94896 \\
\hline Signal transduction activity & 26 & 8.15047 & 0.99997 \\
\hline
\end{tabular}

expression profiles of 1308 lncRNAs determined to be different expression (fold change $\geq 4.0$ or $\leq 0.25, \mathrm{P}<0.01$ ) between $\mathrm{GBM}$ and normal brain samples. Among these, 654 lncRNAs were identified to be upregulated more than four-fold in GBM group compared with that in normal brain group, while 654 lncRNAs were downregulated more than four-fold $(\mathrm{P}<0.01)$.

Potential targets of the differentially expressed IncRNAs are integrated with differentially expressed $m R N A s$. Since IncRNAs regulate the expression of its target genes, the next step is to construct the relationship between the expression profile of the mRNA and predicted target genes of differentially expressed lncRNAs. Procedures for discovering the target genes consisted of two steps. The first step was to predict potential lncRNA targets in the database via target prediction programs. The second step was to integrate the predicted potential lncRNA targets with the different expressed the mRNAs in the profile (fold change $\geq 4$ or $\leq 0.25, \mathrm{P}<0.01$ ). The above methods were 
Table II. Target gene-related pathways.

\begin{tabular}{llll}
\hline Term & Count & P-value & \\
\hline PPAR signaling pathway & 4 & 0.033870 & ACOX1, SLC27A5, CYP8B1, PCK2 \\
Focal adhesion & 5 & 0.284268 & IGF1, MYLPF, FLT4, COMP, MYL2 \\
Regulation of actin cytoskeleton & 5 & 0.330142 & MYLPF, FGF22, APC2, MYL2, BAIAP2 \\
B cell receptor signaling pathway & 2 & 0.388098 & CD79A, CARD11 \\
Calcium signaling pathway & 4 & 0.39238 & GRIN1, TBXA2R, TNNC1, ATP2B3 \\
Tight junction & 3 & 0.419958 & MYLPF, CLDN9, MYL2 \\
mTOR signaling pathway & 1 & 0.60943 & IGF1 \\
Chemokine signaling pathway & 3 & 0.672354 & CCL14, GNG7, GNG13 \\
p53 signaling pathway & 1 & 0.708133 & IGF1 \\
Insulin signaling pathway & 2 & 0.711425 & FBP1, PCK2 \\
MAPK signaling pathway & 4 & 0.717806 & TAOK1, RASGRP4, FGF22, CACNG6 \\
Wnt signaling pathway & 2 & 0.757316 & APC2, DAAM2 \\
Jak-STAT signaling pathway & 2 & 0.770129 & LEPR, CSF3 \\
TGF- $\beta$ signaling pathway & 1 & 0.789934 & COMP \\
Gap junction & 1 & 0.808316 & TUBB4 \\
Toll-like receptor signaling pathway & 1 & 0.840441 & IRF7 \\
T cell receptor signaling pathway & 1 & 0.8597 & CARD11 \\
\hline
\end{tabular}

applied to lncRNA and mRNA expression dataset in one GBM tissue samples and one normal tissue samples with 1,108 differentially expressed mRNAs and 1,308 differentially expressed IncRNAs. This resulted in extremely complex lncRNA target networks. We found 104 matched lncRNA-mRNA pairs for 91 differentially expressed lncRNAs and 84 differentially expressed mRNA.

PPAR pathway might be regulated by IncRNAs according to bioinformatics analyses. GO analysis showed that the high-enrichment GO targeted by both overexpressed and underexpressed lncRNAs were other metabolic processes (Fig. 3 and Table I). The pathway analyses revealed that there were 17 different pathways corresponding to the target genes, one of which was the PPAR signaling pathway in the GBM group compared with the normal brain group with an enrichment $(\mathrm{P}<0.05$, Table II).

Construction of lncRNA:mRNA association networks. Using the statistically significant bij-association scores, we constructed association networks including the differently expressed lncRNAs and target genes (Fig. 4). The resulting lncRNA:mRNA association network had 175 nodes and 104 connections between the 91 lncRNAs and the 84 mRNAs. This network indicated that TBXA2R were predicted to be targets of 31 lncRNAs, GRIN1 were targeted by seven of the lncRNAs, TNFRSF14 were targeted by six of the lncRNAs, and five mRNAs (ACOX1, COMP, CSF3, IGF1 and RASGRP4) were targeted by four of the lncRNAs. Most of the targets mentioned here have been reported to be linked to cancer. For example, high levels of TBXA2R has been previously reported in various tumors including prostate, glioma, melanoma, adenocarcinoma and squamous cell carcinoma of the lung (15). Similarly,
TBXA2R expression has been shown to influence endothelial cell migration and angiogenesis (16). Furthermore, TBXA2R levels are associated with disease-free survival, indicating that the receptor is a prognostic factor in clinical breast cancer (17). ANXA2 up-regulation was reported to be associated with human hepatocellular carcinoma, pancreatic adenocarcinoma, high-grade glioma, gastric carcinoma, acute promyelocytic leukemia and primary colorectal cancer (18).

ASLNC22381 and ASLNC20819 might be the key regulators of glioma pathway in GBM. The IncRNA-mRNA interaction networks related to the pathway we predicted previously are established in Fig. 4. As shown in Fig. 5, the up-regulated lncRNAs ASLNC22381 and ASLNC20819 showed the important position in the glioma pathway. The ASLNC22381 and ASLNC20819 is predicted to target growth factor-related IGF-1 genes, which might play an important role in the recurrence and malignant progression of glioma (19).

\section{Discussion}

GBM is the most common and aggressive type of primary brain tumor in humans, involving glial cells and accounting for $52 \%$ of all parenchymal brain tumor cases and $20 \%$ of all intracranial tumors (20). GBM is located preferentially in the cerebral hemispheres. In the past two decades, the molecular mechanisms to treat GBM have extensively been studied. However, the pathogenesis of this disease is still vague, and the gene regulation involved in this disease has been unclear until now.

Increasing evidence has confirmed lncRNAs to be one of the most important factors controlling gene expression (21). Therefore, we evaluated the lncRNA expression profile in the tissue of GBM patient to reveal the potential role of lncRNAs in 


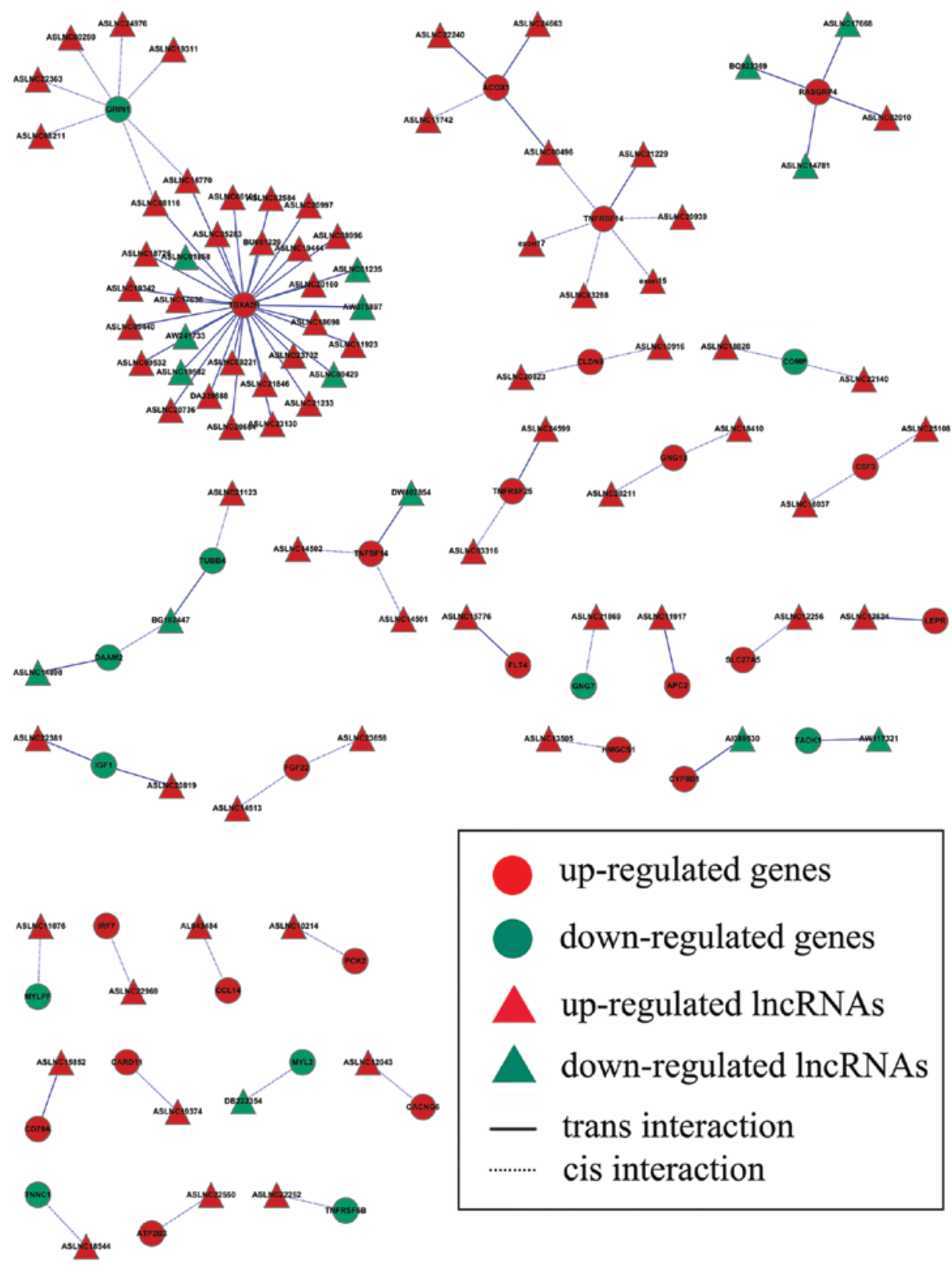

Figure 4. IncRNA-mRNA association network.

the pathogenesis of GBM (Figs. 1 and 2). Microarray techniques revealed a set of differentially expressed lncRNAs, with 654 upregulated and 654 downregulated lncRNAs in GBM tissue when compared to normal brain tissue (fold change $\geq 4$ or $\leq 0.25$, $\mathrm{P}<0.01)$.

Recent studies demonstrate that lncRNAs can guide changes in gene expression either in cis (on neighboring genes) or in trans (distantly located genes) manner that is not easily predicted based on lncRNA sequence $(22,23)$. In principle, IncRNAs can guide chromatin change in cis in a cotranscriptional manner (tethered by RNA polymerase) or as a complementary target for small regulatory RNAs; guidance in trans can occur by lncRNA binding to target DNA as an RNA:DNA heteroduplex, as RNA:DNA:DNA triplex, or RNA recognition of complex surface of specific chromatin features (9).
The role of lncRNAs in disease processes creates an urgency to understand the mechanisms by which these RNAs seek their targets. The earliest lncRNAs suggested a simplistic model where the RNA remains tethered to the site of origin to regulate transcriptional changes in $c i s$. Perhaps the most intensely studied and best understood cis mechanism of regulation by lncRNAs is the mammalian $\mathrm{X}$ inactivation center (Xic), a genetic locus that specifies a number of lncRNAs, including Xist (22). Target genes of cis mechanism of regulation by $\operatorname{lncRNAs}$ were predicted via genome browser and annotation tool.

In contrast to the group of cis-regulatory lncRNAs, there are a couple of examples of lncRNAs that exert their transcriptional effects across chromosomes in trans. Here we have introduced the program RNAplex algorithms which reduces the time needed to localize putative hybridization sites, mainly by 


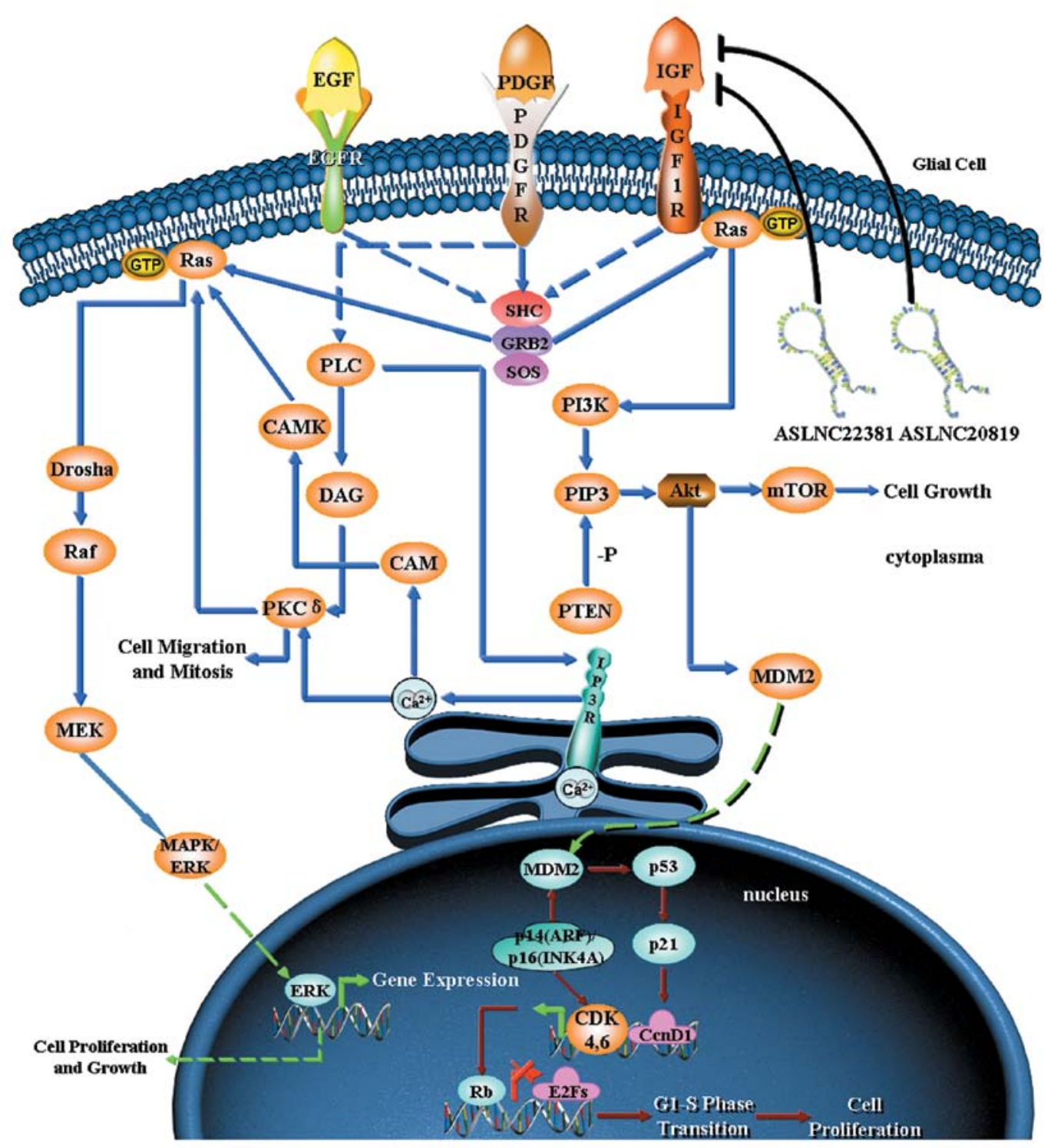

Figure 5. Novel molecular regulation of the glioma signaling pathways through which ASLNC22381 and ASLNC20819 promote recurrence and malignant progression of glioma. This image is a modification of QIAGEN's original, copyrighted image by Lei Han.

neglecting intramolecular interactions and by using a slighlty simplified energy model (12). Combined with, e.g. RNAup we can find high-confidence targets, with only a slight loss of sensitivity. Finally apart from the speed improvement, RNAplex in contrast to similar programs, can recover short, highly stable interactions between two RNAs, by introducing a per nucleotide penalty. As a consequence, RNAplex is well suited for localizing putative lncRNA interactions partners in large amount of genomic data.

To improve the accuracy of target prediction, we further combined differentially expressed mRNA with target prediction of differentially expressed lncRNAs. In order to gain insight into the function of targets of lncRNAs, GO term and KEGG pathway annotation were applied to their target gene pool. KEGG annotation showed a significant change with the PPAR signaling pathway in the GBM group compared with the normal brain group (Fig. 3, and Tables I and II). Further investigation of the lncRNA-gene network showed that the ASLNC22381 and ASLNC20819 was predicted to target growth factor-related IGF-1 genes of the glioma pathway, which might play an important role in the recurrence and malignant progression of glioma (Figs. 4 and 5) (19).

In conclusion, our study revealed for the first time the IncRNAs that are differentially expressed in GBM when compared with the normal brain tissue. Their regulation roles in the PPAR signaling pathways may be involved in the pathogenesis of the GBM. Moreover, ASLNC22381 and ASLNC20819 may have great importance in the glioma pathway via their target IGF-1 and contribute to recurrence and malignant progression of glioma, but confirmation and elucidation of this mechanism requires further study. Present results also point to several exciting directions for future research. Each possible lncRNA-mRNA pair we identified is a strong candidate for a major study to definitively confirm the presence of specific lncRNA-mRNA interactions, thus creating a more detailed picture of the pathogenesis of GBM. 


\section{Acknowledgements}

This study was financially supported by the National Natural Science Foundation of China (81001128, 81172406), Natural Science Foundation of Tianjin Municipal Science and Technology Commission (09JZCD17600, 10SYSYJC28800), Tianjin City High School Science \& Technology Fund (2009CD01, 20090228). The authors wish to thank Shanghai Sensichip Infotech Co., Ltd. (Shanghai, China) for the assistance in bioinformatic analysis.

\section{References}

1. Tzadok S, Beery E, Israeli M, et al: In vitro novel combinations of psychotropics and anti-cancer modalities in U87 human glioblastoma cells. Int J Oncol 4: 1043-1051, 2010.

2. Fiorenzo P, Mongiardi MP, Dimitri D, et al: HIF1-positive and HIF1-negative glioblastoma cells compete in vitro but cooperate in tumor growth in vivo. Int J Oncol 4: 785-791, 2010.

3. Wang Y, Chen L, Bao Z, et al: Inhibition of STAT3 reverses alkylator resistance through modulation of the AKT and $\beta$-catenin signaling pathways. Oncol Rep 5: 1173-1180, 2011.

4. Wang LF, Fokas E, Bieker M, et al: Increased expression of EphA2 correlates with adverse outcome in primary and recurrent glioblastoma multiforme patients. Oncol Rep 1: 151-156, 2008.

5. Dong H, Luo L, Hong S, et al: Integrated analysis of mutations, miRNA and mRNA expression in glioblastoma. BMC Syst Biol 4: 163,2010

6. Mercer TR, Dinger ME and Mattick JS: Long non-coding RNAs: insights into functions. Nat Rev Genet 3: 155-159, 2009.

7. Zong X, Tripathi V and Prasanth KV: RNA splicing control: Yet another gene regulatory role for long nuclear noncoding RNAs. RNA Biol 6: 968-977, 2011.

8. Wang KC and Chang HY: Molecular mechanisms of long noncoding RNAs. Mol Cell 6: 904-914, 2011.

9. Pauli A, Rinn JL and Schier AF: Non-coding RNAs as regulators of embryogenesis. Nat Rev Genet 2: 136-149, 2011.

10. Liao Q, Liu C, Yuan X, et al: Large-scale prediction of long noncoding RNA functions in a coding-non-coding gene co-expression network. Nucleic Acids Res 9: 3864-3878, 2011.
11. Chen L, Li H, Han L, et al: Expression and function of miR-27b in human glioma. Oncol Rep 6: 1617-1621, 2011.

12. Tafer H and Hofacker IL: RNAplex: a fast tool for RNA-RNA interaction search. Bioinformatics 22: 2657-2663, 2008.

13. Dennis G Jr, Sherman BT, Hosack DA, Yang J, Gao W, Lane HC and Lempicki RA: DAVID: Database for Annotation, Visualization, and Integrated Discovery. Genome Biol 5: P3, 2003.

14. Zhang C, Han L, Zhang A, et al: Global changes of mRNA expression reveals an increased activity of the interferon-induced signal transducer and activator of transcription (STAT) pathway by repression of miR-221/222 in glioblastoma U251 cells. Int J Oncol 6: 1503-1512, 2010.

15. Ermert L, Dierkes C and Ermert M: Immunohistochemical expression of cyclooxygenase isoenzymes and downstream enzymes in human lung tumors. Clin Cancer Res 9: 1604-1610, 2003.

16. Nie D, Lamberti M, Zacharek A, et al: Thromboxane A(2) regulation of endothelial cell migration, angiogenesis, and tumor metastasis. Biochem Biophys Res Commun 1: 245-251, 2000.

17. Watkins G, Douglas-Jones A, Mansel RE and Jiang WG: Expression of thromboxane synthase, TBXAS1 and the thromboxane A2 receptor, TBXA2R, in human breast cancer. Int Semin Surg Oncol 2: 23, 2005

18. Bae SM, Min HJ, Ding GH, et al: Protein expression profile using two-dimensional gel analysis in squamous cervical cancer patients. Cancer Res Treat 2: 99-107, 2006.

19. Baritaki S, Chatzinikola AM, Vakis AF, et al: YY1 overexpression in human brain gliomas and meningiomas correlates with TGF-beta1, IGF-1 and FGF-2 mRNA levels. Cancer Invest 2: 184-192, 2009.

20. Wick W, Weller M, Weiler M, Batchelor T, Yung AW and Platten M: Pathway inhibition: emerging molecular targets for treating glioblastoma. Neuro Oncol 6: 566-579, 2011.

21. Khachane AN and Harrison PM: Mining mammalian transcript data for functional long non-coding RNAs. PLoS One 4: E10316, 2010.

22. Hung $\mathrm{T}$ and Chang HY: Long non-coding RNA in genome regulation: prospects and mechanisms. RNA Biol 5: 582-585, 2010.

23. Qureshi IA, Mattick JS and Mehler MF: Long non-coding RNAs in nervous system function and disease. Brain Res 1338: 20-35, 2010. 